\title{
Temporal changes in biological traits of diatom communities in response to an oil spill in a subtropical river
}

\section{DENISE M. DE FARIA, JÚLIO C. COSTIN, PRISCILA I. TREMARIN and THELMAA.V. LUDWIG}

\author{
Programa de Pós-Graduação em Botânica/PGBOT-UFPR, Departamento de Botânica, Setor de Ciências Biológicas, \\ Campus Centro Politécnico, Universidade Federal do Paraná/UFPR, Caixa Postal 19031, 81531-980 Curitiba, PR, Brazil
}

Manuscript received on November 3, 2017; accepted for publication on August 10, 2018

\begin{abstract}
How to cite: FARIA DM, COSTIN JC, TREMARIN PI AND LUDWIG TAV. 2019. Temporal changes in biological traits of diatom communities in response to an oil spill in a subtropical river. An Acad Bras Cienc 91: e20170863. DOI 10.1590/0001-3765201920170863.
\end{abstract}

\begin{abstract}
Diatom growth strategies, which are used as a proxy to analyze disturbances and environmental impacts, can also provide reliable information about environmental gradients. In this study, we used diatom communities and biological traits to assess the structure of epilithic diatoms after an oil spill in a large tributary of the Iguaçu River (Paraná, Brazil). Epilithon and water samples were taken on July 12th, 2004, and after an unexpected oil spill, on the following days: 09/19, 10/03, and 10/13/2004. We observed that the spill caused a distinct shift in both diatom composition and guild group. Diversity decreased and pollution-tolerant diatoms of the motile group became dominant in response to the elevated nutrient levels and reduced light. Tube-forming diatoms, which are strong competitors for light, dominated the epilithon 24 days after the oil spill. Both diatom guilds and growth forms were successfully used to predict the environmental conditions. While diatom guilds responded to disturbances along a temporal gradient, changes inside the guilds were the main factor for understanding the environmental gradient.
\end{abstract}

Key words: diatom guilds, disturbance, diversity, epilithon, growth forms.

\section{INTRODUCTION}

Diatoms are a very diverse group of unicellular photosynthetic microorganisms characterized by a silicified cell wall known as frustule whose ornamentation is used for taxonomy purposes (Round et al. 1990). Diatoms may live free-floating in the water column (phytoplankton) or attached to submerged substrates (periphyton), lying in a polysaccharide matrix with other microorganisms (Wetzel 1983). Specialized structures in the mucilage extruding from the apical pore field, the

Correspondence to: Denise Matias de Faria

E-mail: matiasdefaria.d@gmail.com

ORCid: https://orcid.org/0000-0003-4241-9099 rimoportulae and the raphe enable cells to adhere to surfaces or to slide across them (Round et al. 1990), thus acting as a competitive strategy for resources in the biofilm (Passy 2007). Diatom growth depends on light, water chemistry, temperature (Patrick and Reimer 1966), resource availability, grazing (Lange et al. 2011), and water disturbances (Peterson and Stevenson 1992).

Due to limited mobility, the attached community is exposed to adverse conditions and is thus well-suited to be used worldwide for assessing environmental impacts (Stevenson et al. 1996). Since diatom taxonomy is usually rather complex and time consuming, the use of biological traits in ecological status assessments can provide a more 
efficient alternative. The use of biological traits in predicting environmental-ecological aspects is suitable when species-level identification may be complicated or even impracticable. The application of diatom-based indices requires accurate species identification and is constrained by geographical limitations. In addition, diatom-based indices are calibrated to local information, which prevents their general use (Lobo et al. 2004a, b, StengerKovács et al. 2007). Ecological guilds group species that exploit the same resources within the same ecological preferences, however, showing different adaptations (Simberloff and Dayan 1991). Diatom ecological guilds are strongly related to environmental gradients and respond to disturbance effects and resource availability (Passy 2007, Lange et al. 2011, Berthon et al. 2011, DeNicola and Kelly 2014). Studies have shown that biological traits can be successfully used for the assessment of ecological conditions in rivers (Passy 2007, Rimet and Bouchez 2011, Lange et al. 2011, Schneck and Mello 2012, Stenger-Kovács et al. 2013), lakes (Faria et al. 2015), and wetlands (Riato et al. 2017) at different levels of stress or disturbance.

Accidental oil spills in marine and freshwater affect aquatic biota by disturbing structures essential for vital processes and by causing chemical intoxication (Snow and Scott 1975). Oil discharges can also raise nutrient availability in the water column, favoring eutrophication (Snow and Scott 1975) and reducing light availability for photosynthetic organisms. In addition, losses in periphytic biomass have been recorded as a result of oil exposure (Singh and Gaur 1989, Nayar et al. 2004). On the other hand, the opposite may also occur, since algae biomass can be stimulated by oil (Snow and Scott 1975), as algae grazers can be suppressed (Cubit et al. 1987) by oil toxicity (Scholten and Kuiper 1987), thereby increasing primary production (Bakke and Johnsen 1979). These antagonizing results may reflect the controlled conditions of the studies, which cannot incorporate the multistressor effects of natural environmental conditions. There is increasing interest in the impacts of oil spills on natural benthic conditions, e.g. a study on mangrove benthic communities in French Guiana (Jézéquel et al. 2017). However, the study did not investigate primary producers and the main focus was preparedness due to high risk of oil spills from the Brazilian offshore companies.

The central idea in monitoring of aquatic environments by using biological traits is the possibility of assessing environmental conditions when limited chemical analysis data are available. We assessed the responses of epilithic diatom communities to an unexpected oil spill which occurred in the subtropical freshwater Negro River (in southern Brazil). We used diatom guilds and growth forms as a proxy to estimate the environmental health in each sample. We hypothesized that a temporal gradient was formed after the oil spill and that the discharge affected diatom community.

\section{METHODS}

\section{STUDY AREA}

The Negro River belongs to Iguaçu River basin, located between the cities of Mafra (Santa Catarina State) and Rio Negro (Paraná State) in Brazil. It is the main tributary of the Iguaçu River (SUDERHSA 2007). The river rises at an elevation of $1400 \mathrm{~m}$ and flows $240 \mathrm{~km}$ before emptying into the Iguaçu River.

On September $12^{\text {th }}, 2004$, approximately 120,000 liters of vegetable oil and 60,000 liters of diesel oil spilled into the Negro River after a train accident. The oil leak was controlled only four days after the accident, on September $16^{\text {th }}$, 2004. The public water supply in the region was shut off to prevent the spread of oils and greases in the water distribution network (Military Police Environmental Protection Company 2004). The water supply was fully normalized on September $20^{\text {th }}, 2004$, a week after the accident. 


\section{DATA SAMPLING}

Water and epilithon samples were taken between two extraction points for the public water supply (26 $06^{\prime} 46.6^{\prime \prime} \mathrm{S}$ and 49 47'22.1' W). The first sampling was done on July $12^{\text {th }}, 2004$, before the oil spill. Diatoms were initially being sampled for taxonomic investigation. Since the oil spill occurred about $22 \mathrm{~km}$ upstream from the sampling site (26 $17^{\circ} 16^{\prime \prime S}$ and $49^{\circ} 72^{\prime} 68^{\prime}$ " W), we chose to sample the same location with the aim of understanding the environmental and community changes after the oil spill. Sampling was performed one week after the accident (after1, September $19^{\text {th }}$ ) and then two weeks later (after2, October $3^{\text {th }}$ ). Another sample was taken 24 days after the oil spill (after3, October $13^{\text {th }}$ ).

Water samples were collected for physical and chemical analyses. Nitrate $\left(\mathrm{NO}_{3}^{-}\right)$, nitrite $\left(\mathrm{NO}_{2}^{-}\right)$, phosphate $\left(\mathrm{PO}_{4}^{3-}\right)$, and total solids (TS) were analyzed at "Núcleo de Controle de Qualidade (Universidade Positivo)"; chemical oxygen demand (COD) and biochemical oxygen demand $\left(\mathrm{BOD}_{5}\right)$ were analyzed at "Centro de Pesquisa e Processamento de Alimentos (Universidade Federal do Paraná)" following the methodologies described in APHA (2012). Secchi depth and temperature were measured in situ. Conductivity (cond) was measured with MWA300 and pH with a QUIMIS Q400 Aequipments (Universidade Positivo). The rainfall data (rain) for the sampling period were provided by Instituto das Águas do Paraná.

Epilithon samples were collected from cobbles ( $\mathrm{n}=3$ in each sampling data; cobble size $\approx 8$ to $10 \mathrm{~cm}$ diameter). Biofilm was removed from the substrates by scraping with toothbrushes. After washing, samples were cleaned with $10 \%$ solution of $\mathrm{KMnO}_{4}$ and $\mathrm{HCl}$ (Simonsen 1974, modified by Moreira-Filho and Valente-Moreira 1981). For the preparation of permanent slides Naphrax ${ }^{\circledR}$ (Brunel Microscopes Ltd. U.K.; IR = 1.73) was used as a mounting medium. For the qualitative and quantitative analyses 600 valves were counted (Kobayasi and Mayama 1982). Diatoms were identified according to specific literature, for example, Patrick and Reimer (1966), Krammer and Lange-Bertalot (1986, 1991a, b), Krammer(1997a, b), Metzeltin and Lange-Bertalot (1998, 2007), Metzeltin et al. (2005), Hofmann et al. (2013), Levkov et al. (2013, 2016), and herein we provide the list of diatom taxa of the Negro River and the accompanying metrics. Diatoms were classified into three guilds: motile, high-profile, and lowprofile (adapted from Passy 2007 and Berthon et al. 2011) and into six growth forms: motile, erect, colonial, tube-forming, pioneer, and planktonic (adapted from Rimet and Bouchez 2011, Berthon et al. 2011 and Faria et al. 2015).

\section{DATA ANALYSIS}

Dendrogram of similarity (two-way dendrogram, Euclidean distance) based on the environmental data was used in PC-ORD v6.0 (McCune and Mefford 2011) to investigate nutrient gradients after the spill. Bartlett's test was applied to check homogeneity of variances across samples and KolmogorovSmirnov test to check normality. To describe the diatom community, we used ranking of abundance and calculated diversity indices (Shannon-Weaver) followed by a $t$-test between diversities using PAST software (Hammer et al. 2001). To investigate richness, one-way ANOVA was used followed by the Tukey test in Statistica 7.1 (StatSoft Inc. 2005). We also provide Spearman's correlation between biotic and abiotic data (excluding the sample before the oil spill). To investigate temporal changes in guild and growth form composition in response to oil spill disturbances, Principal Component Analysis (PCA) was performed using data from all the three sampling events after the spill. Data was normalized by $\log \mathrm{x}+1$ transformation. 


\section{RESULTS}

Dendrogram of similarity using environmental data showed that the primary clustering separated the sample taken one week after the oil spill (after1) with $100 \%$ dissimilarity (Fig.1). Sample after1 showed reduced $\mathrm{pH}(6.9)$ and conductivity $(29 \mu \mathrm{S}$ $\left.\mathrm{cm}^{-1}\right)$ and increased TS (100 $\left.\mathrm{mg} \mathrm{L}^{-1}\right)$ values; higher $\mathrm{BOD}_{5}\left(6.29 \mathrm{mg} \mathrm{O}_{2} \mathrm{~L}^{-1}\right), \mathrm{COD}\left(13.4 \mathrm{O}_{2} \mathrm{~L}^{-1}\right), \mathrm{NO}_{3}^{-}$ (1.9 $\left.\mathrm{mg} \mathrm{L}^{-1}\right)$, and $\mathrm{NO}_{2}^{-}\left(0.03 \mathrm{mg} \mathrm{L}^{-1}\right)$ values were also registered. The subsequent samples (after2 and after3) have been grouped together due to high similarity; both showed increases in $\mathrm{pH}$ (7.2) and conductivity $\left(39 \mu \mathrm{S} \mathrm{cm}^{-1}\right)$, as well as high rainfall (200 mm), which kept the TS values high $( \pm 95 \mathrm{mg}$ $\left.\mathrm{L}^{-1}\right) . \mathrm{PO}_{4}^{3-}$ showed a temporal gradient decreasing from after1 $\left(0.47 \mathrm{mg} \mathrm{L}^{-1}\right)$ to after3 $\left(0.14 \mathrm{mg} \mathrm{L}^{-1}\right)$. After 3 showed some increases in water column light penetration $(5 \mathrm{~cm})$ as well as reduced $\mathrm{BOD}_{5}$ (3.7 $\left.\mathrm{mg} \mathrm{O}_{2} \mathrm{~L}^{-1}\right)$ and $\operatorname{COD}\left(5.5 \mathrm{O}_{2} \mathrm{~L}^{-1}\right)$ values; after3 was grouped with the sample before the oil spill, with $\sim 60 \%$ similarity.

A total of ninety-nine diatom taxa were identified, classified into 35 genera and 22 families. The most representative families were Naviculaceae and Cymbelaceae (Table I). Rank abundance revealed that the sample taken before the oil spill had a greater uniformity, while the sample taken after had less. Greater richness and diversity were recorded before the oil spill $\left(\mathrm{S}^{\prime}=77\right.$ and $\mathrm{H}^{\prime}= \pm 3.09$, respectively); the motile guild had the highest richness ( $\left.S^{\prime}=49\right)$, followed by the highprofile $\left(S^{\prime}=23\right)$ and low-profile guilds $\left(S^{\prime}=5\right)$.

We investigated the temporal gradient after the oil spill. Diversity $t$-test showed a negative diversity gradient reducing over time $(t=9.93, p<0.001)$, suggesting changes in diatom community composition as a result of a temporal environmental gradient. We found a richness temporal gradient which declined from after1 $\left(\mathrm{S}^{\prime}=53\right)$ to after3 $\left(\mathrm{S}^{\prime}=34 ; \mathrm{F}=16.80\right.$, $p<0.001)$. In after1, the motile guild presented higher richness $\left(S^{\prime}=28\right)$ than the high-profile $\left(S^{\prime}=21\right)$ and the low-profile $\left(S^{\prime}=4\right)$ guilds; the three guilds

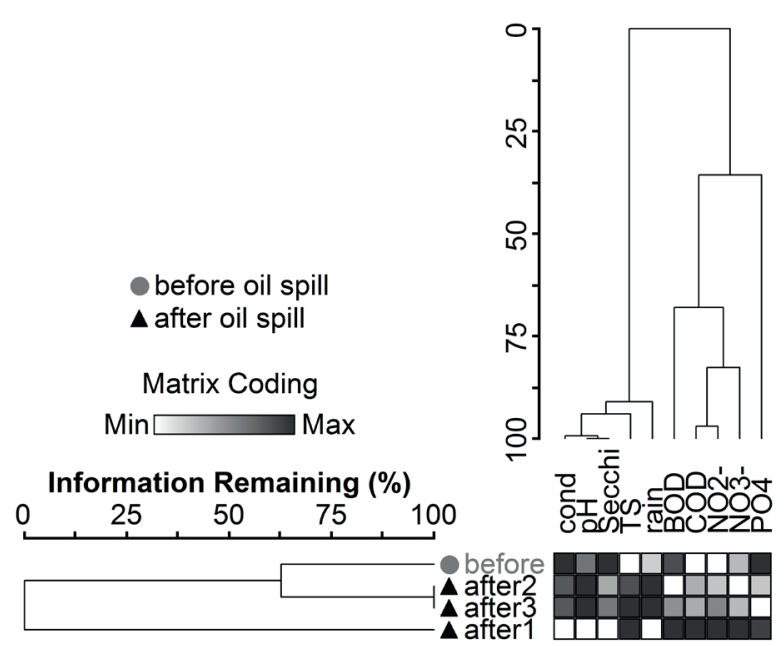

Figure 1 - Dendrogram of similarity showing environmental data before and after the oil spill in Negro River, Brazil.

decreased over time and in after3, richness was as follows: motile guilds $\left(S^{\prime}=16\right)$, high profile guilds $\left(S^{\prime}=15\right)$, and low-profile guilds $\left(S^{\prime}=3\right)$. In after3 we found that $43 \%$ of diatom community relative abundance was comprised of the tube-forming diatom Encyonema perpusillum (Cleve-Euler) and 26\% of the motile diatom Navigeia aikenensis (R.M. Patrick) L.N. Bukhtiyarova.

Higher motile guild diversity was registered before the oil spill $\left(\mathrm{H}^{\prime}=3.06\right)$. Diversity $t$-tests showed that the guild diversity was lower in after 1 $\left(\mathrm{H}^{\prime}=1.9 ; t=9.70, p<0.001\right)$ due to the abundance of Navigeia aikenensis and Navicula veneta Kützing, as well as Mayamaea permitis (Hustedt) K. Bruder \& Medlin and Sellaphora nigri (De Notaris) C.E. Wetzel \& L. Ector. The motile guild, which dominated the diatom community in after 1 (70\%) was positively correlated with phosphate concentration in water $(r=0.99, p=0.03)$. The proportion of motile guild abundance decreased over time $(\mathrm{F}=17.65, p<0.001)$ due to replacement by the low-profile guild. Moreover, the motile diatoms Luticula goeppertiana (Bleisch) D.G. Mann and N. aikenensis persisted in the periphytic matrix after the oil spill, whereas L. goeppertiana grew between after 1 and after 2 , while $N$. aikenensis was observed in the periphytic matrix until after3. 
TABLE I

Diatoms from Negro River, classification and metrics.

\begin{tabular}{|c|c|c|c|c|c|c|}
\hline \multirow[b]{2}{*}{ Taxa } & \multirow[b]{2}{*}{$\begin{array}{c}\text { Growth form } \\
\text { and ecological } \\
\text { guild }\end{array}$} & \multicolumn{5}{|c|}{ Metric } \\
\hline & & Lenght $(\mu \mathrm{m})$ & Width $(\mu \mathrm{m})$ & Striae $(10 \mu \mathrm{m})$ & $\begin{array}{l}\text { Areolae }(10 \\
\mu \mathrm{m})\end{array}$ & $\begin{array}{c}\text { Fibulae/ } \\
\text { Costae/Alar } \\
\text { canals }(10 \\
\mu \mathrm{m})\end{array}$ \\
\hline \multicolumn{7}{|l|}{ Stephanodiscaceae } \\
\hline $\begin{array}{l}\text { Discostella stelligera } \\
\text { (Cleve \& Grunow) } \\
\text { Houk \& Klee }\end{array}$ & $\begin{array}{l}\text { Planktonic; } \\
\text { high-profile }\end{array}$ & $\ldots \ldots .$. & $6-9$ & $15-16$ & ........ & \\
\hline \multicolumn{7}{|l|}{ Melosiraceae } \\
\hline $\begin{array}{l}\text { Melosira varians } \mathrm{C} . \\
\text { Agardh }\end{array}$ & $\begin{array}{l}\text { Planktonic; } \\
\text { high-profile }\end{array}$ & $12-23.2$ & $16.8-25.3$ & $\ldots \ldots \ldots$ & $\ldots \ldots \ldots$ & \\
\hline \multicolumn{7}{|l|}{ Aulacoseiraceae } \\
\hline $\begin{array}{l}\text { Aulacoseira ambigua } \\
\text { (Grunow) Simonsen }\end{array}$ & $\begin{array}{l}\text { Planktonic; } \\
\text { high-profile }\end{array}$ & $16.3-25$ & $5-8.4$ & $14-18$ & $16-18$ & \\
\hline $\begin{array}{c}\text { Aulacoseira pusilla (F. } \\
\text { Meister) A. Tuji \& A. } \\
\text { Houki }\end{array}$ & $\begin{array}{l}\text { Planktonic; } \\
\text { high-profile }\end{array}$ & $6.3-8$ & $6-7$ & $18-20$ & 20 & \\
\hline $\begin{array}{l}\text { Aulacoseira tenella } \\
\text { (Nygaard) Simonsen }\end{array}$ & $\begin{array}{l}\text { Planktonic; } \\
\text { high-profile }\end{array}$ & $3.1-3.5$ & $5.8-9$ & $18-22$ & $15-16$ & \\
\hline \multicolumn{7}{|l|}{ Orthoseiraceae } \\
\hline $\begin{array}{l}\text { Orthoseira dentroteres } \\
\text { (Ehrenberg) Crawford }\end{array}$ & $\begin{array}{l}\text { Planktonic; } \\
\text { high-profile }\end{array}$ & $\ldots \ldots \ldots$ & $9-14.7$ & $17-18$ & 18 & \\
\hline \multicolumn{7}{|l|}{ Fragilariaceae } \\
\hline $\begin{array}{c}\text { Fragilaria } \\
\text { fragilarioides (Grunow) } \\
\text { Cholnoky }\end{array}$ & $\begin{array}{l}\text { Erect; high- } \\
\text { profile }\end{array}$ & $26.8-46.8$ & $3.7-4.2$ & $13-15$ & ......... & \\
\hline $\begin{array}{l}\text { Fragilaria rumpens } \\
\text { (Kützing) G.W.F. } \\
\text { Carlson }\end{array}$ & $\begin{array}{l}\text { Erect; high- } \\
\text { profile }\end{array}$ & $22-35$ & $3-4$ & $18-20$ & ......... & \\
\hline $\begin{array}{c}\text { Staurosirella } \\
\text { leptostauron } \\
\text { (Ehrenberg) D.G. } \\
\text { Williams \& Round var. } \\
\text { leptostauron }\end{array}$ & $\begin{array}{l}\text { Colonial; } \\
\text { high-profile }\end{array}$ & $14-20$ & $5-7$ & $6-8$ & $\ldots \ldots$. & \\
\hline $\begin{array}{l}\text { Staurosirella pinnata } \\
\text { (Ehrenberg) D.G. } \\
\text { Williams \& Round }\end{array}$ & $\begin{array}{l}\text { Colonial; } \\
\text { high-profile }\end{array}$ & $4.2-9.4$ & $3-5$ & $13-16$ & $\ldots \ldots \ldots$ & \\
\hline \multicolumn{7}{|l|}{ Eunotiaceae } \\
\hline $\begin{array}{c}\text { Eunotia intermedia } \\
\text { (Krasske ex Hustedt) } \\
\text { Nörpel \& Lange- } \\
\text { Bertalot }\end{array}$ & $\begin{array}{l}\text { Colonial; } \\
\text { high-profile }\end{array}$ & $11-15$ & $3-4$ & $13-14$ & $\ldots \ldots \ldots$ & \\
\hline $\begin{array}{l}\text { Eunotia minor } \\
\text { (Kützing) Grunow }\end{array}$ & $\begin{array}{c}\text { Colonial; } \\
\text { high-profile }\end{array}$ & $22.6-38$ & $4-6$ & $15-16$ & $\ldots \ldots .$. & \\
\hline
\end{tabular}


TABLE I (continuation)

\begin{tabular}{|c|c|c|c|c|c|c|}
\hline \multirow[b]{2}{*}{ Taxa } & \multirow[b]{2}{*}{$\begin{array}{l}\text { Growth form } \\
\text { and ecological } \\
\text { guild }\end{array}$} & \multicolumn{5}{|c|}{ Metric } \\
\hline & & Lenght $(\mu \mathrm{m})$ & Width $(\mu \mathrm{m})$ & Striae $(10 \mu \mathrm{m})$ & $\begin{array}{c}\text { Areolae }(10 \\
\mu \mathrm{m})\end{array}$ & $\begin{array}{c}\text { Fibulae/ } \\
\text { Costae/Alar } \\
\text { canals }(10 \\
\mu \mathrm{m}) \\
\end{array}$ \\
\hline $\begin{array}{l}\text { Eunotia rabenhorstiana } \\
\text { (Grunow) Hustedt }\end{array}$ & $\begin{array}{l}\text { Colonial; } \\
\text { high-profile }\end{array}$ & $84-133$ & $6-8$ & $14-17$ & $\ldots \ldots$. & \\
\hline $\begin{array}{c}\text { Eunotia rabenhorstii } \\
\text { Cleve \& Grunow }\end{array}$ & $\begin{array}{c}\text { Colonial; } \\
\text { high-profile }\end{array}$ & $16-27$ & $6-8$ & $12-14$ & $\ldots \ldots \ldots$ & \\
\hline Eunotia siolii Hustedt & $\begin{array}{c}\text { Colonial; } \\
\text { high-profile }\end{array}$ & $19-22$ & $4-5$ & $8-10$ & $\ldots \ldots .$. & \\
\hline $\begin{array}{l}\text { Eunotia sudetica } \mathrm{O} . \\
\text { Müller }\end{array}$ & $\begin{array}{l}\text { Colonial; } \\
\text { high-profile }\end{array}$ & $35-39$ & $6-7$ & $10-12$ & ......... & \\
\hline \multicolumn{7}{|l|}{ Cymbellaceae } \\
\hline $\begin{array}{l}\text { Adlafia drouetiana } \\
\text { (R.M. Patrick) } \\
\text { Metzeltin \& Lange- } \\
\text { Bertalot }\end{array}$ & $\begin{array}{l}\text { Motile; motile } \\
\text { guild }\end{array}$ & $15.3-15.7$ & $3.6-4.2$ & $24-26$ & $\cdots \cdots$ & \\
\hline $\begin{array}{c}\text { Adlafia muscora } \\
\text { (Kociolek \& Reviers) } \\
\text { G. Moser, Lange- } \\
\text { Bertalot \& Metzeltin }\end{array}$ & $\begin{array}{l}\text { Motile; motile } \\
\text { guild }\end{array}$ & $14-15$ & $3-4$ & $26-28$ & ......... & \\
\hline $\begin{array}{l}\text { Encyonema minutum } \\
\text { (Hilse) Mann }\end{array}$ & $\begin{array}{l}\text { Tube-forming; } \\
\text { high-profile }\end{array}$ & $12-20$ & $4-5.2$ & $12-14$ & ......... & \\
\hline $\begin{array}{l}\text { Encyonema perpusillum } \\
\text { (Cleve) D.G. Mann }\end{array}$ & $\begin{array}{l}\text { Tube-forming; } \\
\text { high-profile }\end{array}$ & $11-20$ & $3.6-4.7$ & $11-12$ & ......... & \\
\hline $\begin{array}{c}\text { Encyonema exuberans } \\
\text { Tremarin, C.E. Wetzel, } \\
\text { T. Ludwig }\end{array}$ & $\begin{array}{l}\text { Tube-forming; } \\
\text { high-profile }\end{array}$ & $18.4-36$ & $5.7-6.3$ & $8-12$ & ........ & \\
\hline $\begin{array}{l}\text { Encyonema silesiacum } \\
\text { (Bleisch) D.G. Mann }\end{array}$ & $\begin{array}{l}\text { Tube-forming; } \\
\text { high-profile }\end{array}$ & $25-31$ & $6-7.8$ & $12-14$ & ......... & \\
\hline $\begin{array}{l}\text { Placoneis clementis } \\
\text { (Grunow) E.J. Cox }\end{array}$ & $\begin{array}{l}\text { Motile; motile } \\
\text { guild }\end{array}$ & $24-35$ & $10-13$ & $12-15$ & ......... & \\
\hline $\begin{array}{c}\text { Placoneis disparilis } \\
\text { (Hustedt) Metzeltin \& } \\
\text { Lange-Bertalot }\end{array}$ & $\begin{array}{l}\text { Motile; motile } \\
\text { guild }\end{array}$ & $36.3-56$ & $14-15.7$ & $12-13$ & $18-20$ & \\
\hline $\begin{array}{c}\text { Placoneis itamoemae } \\
\text { Straube, Tremarin \& T. } \\
\text { Ludwig }\end{array}$ & $\begin{array}{l}\text { Motile; motile } \\
\text { guild }\end{array}$ & $26.3-35$ & $13.6-14$ & $14-18$ & & \\
\hline $\begin{array}{l}\text { Placoneis porifera var. } \\
\text { opportuna (Hustedt) E. } \\
\text { Novelo, R. Tavera \& C. } \\
\text { Ibarra }\end{array}$ & $\begin{array}{l}\text { Motile; motile } \\
\text { guild }\end{array}$ & $13.1-16.3$ & $6.8-7.3$ & $12-14$ & $\ldots \ldots \ldots$ & \\
\hline \multicolumn{7}{|l|}{ Gomphonemataceae } \\
\hline $\begin{array}{c}\text { Gomphonema costei } \\
\text { Metzeltin \& Lange- } \\
\text { Bertalot }\end{array}$ & $\begin{array}{l}\text { Erect; high- } \\
\text { profile }\end{array}$ & $23.6-45$ & $6.8-7.8$ & $7-9$ & ......... & \\
\hline $\begin{array}{c}\text { Gomphonema } \\
\text { exilissimum (Grunow) } \\
\text { Lange-Bertlot \& E. } \\
\text { Reichardt }\end{array}$ & $\begin{array}{l}\text { Erect; high- } \\
\text { profile }\end{array}$ & $15.2-28.4$ & $4.2-4.7$ & $14-16$ & ........ & \\
\hline
\end{tabular}


TABLE I (continuation)

\begin{tabular}{|c|c|c|c|c|c|c|}
\hline \multirow[b]{2}{*}{ Taxa } & \multirow[b]{2}{*}{$\begin{array}{l}\text { Growth form } \\
\text { and ecological } \\
\text { guild }\end{array}$} & \multicolumn{5}{|c|}{ Metric } \\
\hline & & Lenght $(\mu \mathrm{m})$ & Width $(\mu \mathrm{m})$ & Striae $(10 \mu \mathrm{m})$ & $\begin{array}{c}\text { Areolae }(10 \\
\mu \mathrm{m})\end{array}$ & $\begin{array}{c}\text { Fibulae/ } \\
\text { Costae/Alar } \\
\text { canals }(10 \\
\mu \mathrm{m})\end{array}$ \\
\hline $\begin{array}{c}\text { Gomphonema gracile } \\
\text { Ehrenberg }\end{array}$ & $\begin{array}{l}\text { Erect; high- } \\
\text { profile }\end{array}$ & $55-63$ & $8-9$ & $10-12$ & ......... & \\
\hline $\begin{array}{c}\text { Gomphonema lagenula } \\
\text { Kützing }\end{array}$ & $\begin{array}{l}\text { Erect; high- } \\
\text { profile }\end{array}$ & $16-22$ & $4-5$ & $11-12$ & ......... & \\
\hline $\begin{array}{c}\text { Gomphonema } \\
\text { mexicanum Grunow }\end{array}$ & $\begin{array}{l}\text { Erect; high- } \\
\text { profile }\end{array}$ & $26.3-58.4$ & $8.4-12$ & $10-12$ & 20 & \\
\hline $\begin{array}{l}\text { Gomphonema parvulum } \\
\text { (Kützing) Kützing }\end{array}$ & $\begin{array}{l}\text { Erect; high- } \\
\text { profile }\end{array}$ & $14-20.5$ & $3.6-4.7$ & $13-15$ & ......... & \\
\hline $\begin{array}{c}\text { Gomphonema } \\
\text { pseudoaugur Lange- } \\
\text { Bertalot }\end{array}$ & $\begin{array}{l}\text { Erect; high- } \\
\text { profile }\end{array}$ & $23.1-32.6$ & 7.8 & $11-14$ & ......... & \\
\hline $\begin{array}{l}\text { Gomphonema pumilum } \\
\text { (Grunow) E. Reichardt } \\
\text { \& Lange-Bertalot }\end{array}$ & $\begin{array}{l}\text { Erect; high- } \\
\text { profile }\end{array}$ & $10-23.1$ & $3.1-4.2$ & $12-13$ & ......... & \\
\hline \multicolumn{7}{|l|}{ Cocconeidaceae } \\
\hline $\begin{array}{l}\text { Cocconeis fluviatilis } \\
\text { J.H. Wallace }\end{array}$ & $\begin{array}{l}\text { Adnate; low- } \\
\text { profile }\end{array}$ & $20-23$ & $10-12$ & $\begin{array}{c}18-20 \mathrm{c} / \mathrm{r} ; 12- \\
13 \mathrm{~s} / \mathrm{r}\end{array}$ & $20 \mathrm{c} / \mathrm{r} ; 10-11 \mathrm{~s} / \mathrm{r}$ & \\
\hline $\begin{array}{c}\text { Cocconeis placentula } \\
\text { var. lineata (Ehrenberg) } \\
\text { Van Heurck }\end{array}$ & $\begin{array}{l}\text { Adnate; low- } \\
\text { profile }\end{array}$ & $30-38$ & $23-29$ & $\begin{array}{c}18 \mathrm{c} / \mathrm{r} ; 18-20 \\
\mathrm{~s} / \mathrm{r}\end{array}$ & $\begin{array}{c}18 \mathrm{c} / \mathrm{r} ; 13-15 \\
\mathrm{~s} / \mathrm{r}\end{array}$ & \\
\hline \multicolumn{7}{|l|}{ Achnanthidiaceae } \\
\hline $\begin{array}{l}\text { Achnanthidium } \\
\text { eutrophilum (Lange- } \\
\text { Bertalot) Lange- } \\
\text { Bertalot }\end{array}$ & $\begin{array}{l}\text { Adnate; low- } \\
\text { profile }\end{array}$ & $10-16$ & $4-5$ & $\begin{array}{c}20 \mathrm{c} / \mathrm{r} ; 24-26 \\
\mathrm{~s} / \mathrm{r}\end{array}$ & ......... & \\
\hline $\begin{array}{l}\text { Achnanthidium exiguum } \\
\text { (Grunow) Czarnecki }\end{array}$ & $\begin{array}{l}\text { Adnate; low- } \\
\text { profile }\end{array}$ & $9-13$ & $5-6$ & $\begin{array}{c}26 \mathrm{c} / \mathrm{r} ; 20-22 \\
\mathrm{~s} / \mathrm{r}\end{array}$ & ......... & \\
\hline $\begin{array}{c}\text { Achnanthidium } \\
\text { minutissimum (Kützing) } \\
\text { Czarnecki }\end{array}$ & $\begin{array}{l}\text { Erect; low- } \\
\text { profile }\end{array}$ & $9-19$ & $3-4$ & ......... & ........ & \\
\hline $\begin{array}{l}\text { Karayevia oblongella } \\
\text { (Oestrup) Aboal }\end{array}$ & $\begin{array}{l}\text { Motile; motile } \\
\text { guild }\end{array}$ & $8-16$ & $5-6$ & $12-4 \mathrm{~s} / \mathrm{r}$ & $\ldots \ldots \ldots$ & \\
\hline $\begin{array}{c}\text { Planothidium } \\
\text { bagualensis C.E. Wetzel } \\
\text { \& L. Ector }\end{array}$ & $\begin{array}{l}\text { Erect; high- } \\
\text { profile }\end{array}$ & $12-23$ & $6-9$ & $\begin{array}{c}16-17 \mathrm{c} / \mathrm{r} ; 11- \\
14 \mathrm{~s} / \mathrm{r}\end{array}$ & .......... & \\
\hline $\begin{array}{c}\text { Planothidium } \\
\text { biporomum (M.H. Hohn } \\
\text { \& Hellermann) Lange- } \\
\text { Bertalot }\end{array}$ & $\begin{array}{l}\text { Erect; high- } \\
\text { profile }\end{array}$ & $16-22$ & $6-8$ & $12-14$ & $\ldots \ldots \ldots$ & \\
\hline $\begin{array}{l}\text { Planothidium dubium } \\
\text { (Grunow) Round \& } \\
\text { L.N. Bukhtiyarova }\end{array}$ & $\begin{array}{l}\text { Erect; high- } \\
\text { profile }\end{array}$ & $16-18$ & $6-7$ & $12-14$ & ......... & \\
\hline $\begin{array}{c}\text { Planothidium } \\
\text { heteromorphum } \\
\text { (Grunow) Lange- } \\
\text { Bertalot }\end{array}$ & $\begin{array}{l}\text { Erect; high- } \\
\text { profile }\end{array}$ & $26-31$ & $13-14$ & $\begin{array}{c}10-11 \mathrm{c} / \mathrm{r} ; 9-10 \\
\mathrm{~s} / \mathrm{r}\end{array}$ & .......... & \\
\hline
\end{tabular}


TABLE I (continuation)

\begin{tabular}{|c|c|c|c|c|c|c|}
\hline \multirow[b]{2}{*}{ Taxa } & \multirow[b]{2}{*}{$\begin{array}{l}\text { Growth form } \\
\text { and ecological } \\
\text { guild }\end{array}$} & \multicolumn{5}{|c|}{ Metric } \\
\hline & & Lenght $(\mu \mathrm{m})$ & Width $(\mu \mathrm{m})$ & Striae $(10 \mu \mathrm{m})$ & $\begin{array}{c}\text { Areolae }(10 \\
\mu \mathrm{m})\end{array}$ & $\begin{array}{c}\text { Fibulae/ } \\
\text { Costae/Alar } \\
\text { canals }(10 \\
\mu \mathrm{m})\end{array}$ \\
\hline $\begin{array}{l}\text { Planothidium rostratum } \\
\text { (Oestrup) Round \& } \\
\text { Bukhtyiarova }\end{array}$ & $\begin{array}{l}\text { Erect; high- } \\
\text { profile }\end{array}$ & $9.4-17$ & $4.2-5.8$ & $11-12$ & $\ldots \ldots \ldots$ & \\
\hline \multicolumn{7}{|l|}{ Diadesmidaceae } \\
\hline $\begin{array}{l}\text { Humidophila contenta } \\
\text { (Grunow) Lowe, } \\
\text { Kociolek, J.R. } \\
\text { Johansen, Van de } \\
\text { Vijver, Lange-Bertalot } \\
\text { \& Kopalová }\end{array}$ & $\begin{array}{c}\text { Colonial; } \\
\text { high-profile }\end{array}$ & $7-11$ & 3 & ........ & $\ldots \ldots .$. & \\
\hline $\begin{array}{l}\text { Luticola goeppertiana } \\
\text { (Bleisch) D.G. Mann ex } \\
\text { J. Rarick, S. Wu, S.S. } \\
\text { Lee \& Edlund }\end{array}$ & $\begin{array}{l}\text { Motile; motile } \\
\text { guild }\end{array}$ & $22-31.5$ & $5.7-8.4$ & $20-22$ & 24 & \\
\hline $\begin{array}{c}\text { Luticola kotschyi } \\
\text { (Grunow) D.G. Mann }\end{array}$ & $\begin{array}{l}\text { Motile; motile } \\
\text { guild }\end{array}$ & $15-23$ & $4.7-7$ & $20-25$ & 26 & \\
\hline $\begin{array}{c}\text { Luticola mutica } \\
\text { (Kützing) D.G. Mann }\end{array}$ & $\begin{array}{l}\text { Motile; motile } \\
\text { guild }\end{array}$ & $12.6-20$ & $5.2-6.3$ & $18-20$ & 22 & \\
\hline $\begin{array}{l}\text { Luticola saxophila (W. } \\
\text { Bock ex Hustedt) D.G. } \\
\text { Mann }\end{array}$ & $\begin{array}{l}\text { Motile; motile } \\
\text { guild }\end{array}$ & $8.9-10$ & 5.7 & $18-22$ & $18-22$ & \\
\hline \multicolumn{7}{|l|}{ Brachysiraceae } \\
\hline $\begin{array}{c}\text { Brachysira brebissonii } \\
\text { R. Ross }\end{array}$ & $\begin{array}{l}\text { Motile; motile } \\
\text { guild }\end{array}$ & $17-23$ & $5-7$ & 20 & $\ldots \ldots \ldots$ & \\
\hline $\begin{array}{l}\text { Brachysira vitrea } \\
\text { (Grunow) R. Ross }\end{array}$ & $\begin{array}{l}\text { Motile; motile } \\
\text { guild }\end{array}$ & $18-26$ & $5-6$ & ......... & $\ldots \ldots \ldots$ & \\
\hline $\begin{array}{l}\text { Nupela praecipuoides } \\
\text { Tremarin \& T. Ludwig }\end{array}$ & $\begin{array}{l}\text { Motile; motile } \\
\text { guild }\end{array}$ & $9-14.7$ & $4.2-4.7$ & ........ & $\ldots \ldots \ldots$ & \\
\hline \multicolumn{7}{|l|}{ Amphipleuraceae } \\
\hline $\begin{array}{c}\text { Frustulia crassinervia } \\
\text { (Brébisson ex W. Smith) } \\
\text { Lange-Bertalot \& } \\
\text { Krammer }\end{array}$ & $\begin{array}{l}\text { Motile; motile } \\
\text { guild }\end{array}$ & 48.4 & 10 & ........ & $\ldots \ldots \ldots$ & \\
\hline $\begin{array}{c}\text { Frustulia neomundana } \\
\text { Lange-Bertalot \& U. } \\
\text { Rumrich }\end{array}$ & $\begin{array}{l}\text { Motile; motile } \\
\text { guild }\end{array}$ & $37-39$ & $8-9$ & ........ & ........ & \\
\hline $\begin{array}{l}\text { Frustulia pumilio } \\
\text { Lange-Bertalot \& U. } \\
\text { Rumrich }\end{array}$ & $\begin{array}{l}\text { Motile; motile } \\
\text { guild }\end{array}$ & $14.7-17.3$ & $4.2-5$ & ......... & & \\
\hline $\begin{array}{c}\text { Frustulia saxonica } \\
\text { Rabenhorst }\end{array}$ & $\begin{array}{l}\text { Motile; motile } \\
\text { guild }\end{array}$ & $42-66$ & $11-14$ & ........ & -....... & \\
\hline $\begin{array}{l}\text { Frustulia vulgaris } \\
\text { (Thwaites) De Toni }\end{array}$ & $\begin{array}{l}\text { Motile; motile } \\
\text { guild }\end{array}$ & $51-54$ & $10-11$ & $\ldots \ldots \ldots$ & $\ldots \ldots .$. & \\
\hline
\end{tabular}


TABLE I (continuation)

\begin{tabular}{|c|c|c|c|c|c|c|}
\hline \multirow[b]{2}{*}{ Taxa } & \multirow[b]{2}{*}{$\begin{array}{l}\text { Growth form } \\
\text { and ecological } \\
\text { guild }\end{array}$} & \multicolumn{5}{|c|}{ Metric } \\
\hline & & Lenght $(\mu \mathrm{m})$ & Width $(\mu \mathrm{m})$ & Striae $(10 \mu \mathrm{m})$ & $\begin{array}{c}\text { Areolae }(10 \\
\mu \mathrm{m})\end{array}$ & $\begin{array}{c}\text { Fibulae/ } \\
\text { Costae/Alar } \\
\text { canals }(10 \\
\mu \mathrm{m}) \\
\end{array}$ \\
\hline \multicolumn{7}{|l|}{ Neidiaceae } \\
\hline $\begin{array}{l}\text { Neidium alpinum } \\
\text { Hustedt }\end{array}$ & $\begin{array}{l}\text { Motile; motile } \\
\text { guild }\end{array}$ & $13.1-35$ & $3.7-4.7$ & ......... & ........ & \\
\hline $\begin{array}{c}\text { Neidium affine } \\
\text { (Ehrenberg) Pfitzer var. } \\
\text { affine }\end{array}$ & $\begin{array}{l}\text { Motile; motile } \\
\text { guild }\end{array}$ & $26.8-50$ & $7.3-11$ & 20 & $\ldots \ldots \ldots$ & \\
\hline $\begin{array}{l}\text { Neidium ampliatum } \\
\text { (Ehrenberg) Krammer }\end{array}$ & $\begin{array}{l}\text { Motile; motile } \\
\text { guild }\end{array}$ & $62-71$ & $15-16$ & $20-22$ & 18 & \\
\hline \multicolumn{7}{|l|}{ Sellaphoraceae } \\
\hline $\begin{array}{c}\text { Sellaphora manguinii } \\
\text { C.E. Wetzel }\end{array}$ & $\begin{array}{l}\text { Motile; motile } \\
\text { guild }\end{array}$ & $10-11$ & 4 & 18 & ....... & \\
\hline $\begin{array}{c}\text { Sellaphora nigri (De } \\
\text { Notaris) C.E. Wetzel \& } \\
\text { L. Ector }\end{array}$ & $\begin{array}{l}\text { Motile; motile } \\
\text { guild }\end{array}$ & $6.8-10.5$ & $2.6-3.1$ & $24-26$ & ........ & \\
\hline $\begin{array}{c}\text { Sellaphora } \\
\text { pseudoarvensis } \\
\text { (Hustedt) C.E. Wetzel \& } \\
\text { L. Ector }\end{array}$ & $\begin{array}{l}\text { Motile; motile } \\
\text { guild }\end{array}$ & 7.3-8.9 & 2.1 & ........ & $\ldots \ldots$. & \\
\hline $\begin{array}{l}\text { Sellaphora saugerresii } \\
\text { (Desmazières) C.E. } \\
\text { Wetzel \& D.G. Mann }\end{array}$ & $\begin{array}{l}\text { Motile; motile } \\
\text { guild }\end{array}$ & $8.4-13.1$ & $3.1-3.6$ & $20-22$ & ......... & \\
\hline $\begin{array}{l}\text { Sellaphora tridentula } \\
\text { (Krasske) C.E. Wetzel }\end{array}$ & $\begin{array}{l}\text { Motile; motile } \\
\text { guild }\end{array}$ & $12-14$ & 3 & $\ldots \ldots$. & ......... & \\
\hline $\begin{array}{c}\text { Sellaphora } \\
\text { ventralochilensis C.E. } \\
\text { Wetzel \& L. Ector }\end{array}$ & $\begin{array}{l}\text { Motile; motile } \\
\text { guild }\end{array}$ & $12-18$ & $4.7-6$ & 24 & ......... & \\
\hline \multicolumn{7}{|l|}{ Pinnulariaceae } \\
\hline $\begin{array}{c}\text { Caloneis hyalina } \\
\text { Hustedt }\end{array}$ & $\begin{array}{l}\text { Motile; motile } \\
\text { guild }\end{array}$ & $13.1-20$ & $3.6-4.7$ & ......... & ......... & \\
\hline $\begin{array}{c}\text { Chamaepinnularia } \\
\text { submuscicola (Krasske) } \\
\text { Lange-Bertalot }\end{array}$ & $\begin{array}{l}\text { Motile; motile } \\
\text { guild }\end{array}$ & $6-12.1$ & $2.6-3.1$ & 18 & $\ldots \ldots \ldots$ & \\
\hline $\begin{array}{l}\text { Pinnularia brauniana } \\
\text { (Grunow) Studnicka }\end{array}$ & $\begin{array}{l}\text { Motile; motile } \\
\text { guild }\end{array}$ & $32-39$ & $5.2-8$ & $11-12$ & $\ldots \ldots \ldots$ & \\
\hline $\begin{array}{l}\text { Pinnularia } \\
\text { microstauron var. } \\
\text { rostrata } \text { Krammer }\end{array}$ & $\begin{array}{l}\text { Motile; motile } \\
\text { guild }\end{array}$ & $20.5-25.2$ & $3.4-4.2$ & 16 & $\ldots \ldots$. & \\
\hline \multicolumn{7}{|l|}{ Naviculaceae } \\
\hline $\begin{array}{c}\text { Hippodonta capitata } \\
\text { subsp. iberoamericana } \\
\text { Metzeltin, Lange- } \\
\text { Bertalot \& García- } \\
\text { Rodríguez }\end{array}$ & $\begin{array}{l}\text { Motile; motile } \\
\text { guild }\end{array}$ & $17.8-20.5$ & 4.7 & $5-6$ & $\ldots \ldots \ldots$ & \\
\hline
\end{tabular}


TABLE I (continuation)

\begin{tabular}{|c|c|c|c|c|c|c|}
\hline \multirow[b]{2}{*}{ Taxa } & \multirow[b]{2}{*}{$\begin{array}{l}\text { Growth form } \\
\text { and ecological } \\
\text { guild }\end{array}$} & \multicolumn{5}{|c|}{ Metric } \\
\hline & & Lenght $(\mu \mathrm{m})$ & Width $(\mu \mathrm{m})$ & Striae $(10 \mu \mathrm{m})$ & $\begin{array}{c}\text { Areolae }(10 \\
\mu \mathrm{m})\end{array}$ & $\begin{array}{c}\text { Fibulae/ } \\
\text { Costae/Alar } \\
\text { canals }(10 \\
\mu \mathrm{m}) \\
\end{array}$ \\
\hline $\begin{array}{c}\text { Mayamaea permitis } \\
\text { (Hustedt) K. Bruder \& } \\
\text { Medlin }\end{array}$ & $\begin{array}{l}\text { Motile; motile } \\
\text { guild }\end{array}$ & $6.8-8$ & $2.6-4$ & $22-24$ & $\ldots \ldots \ldots$ & \\
\hline $\begin{array}{c}\text { Navicula cryptocephala } \\
\text { Kützing }\end{array}$ & $\begin{array}{l}\text { Motile; motile } \\
\text { guild }\end{array}$ & $26-39$ & $4.7-5.7$ & $14-15$ & -....... & \\
\hline $\begin{array}{c}\text { Navicula cryptotenella } \\
\text { Lange-Bertalot }\end{array}$ & $\begin{array}{l}\text { Motile; motile } \\
\text { guild }\end{array}$ & $15.2-23$ & $4.7-5.2$ & $16-18$ & $\cdots \cdots \cdots$ & \\
\hline $\begin{array}{c}\text { Navicula erifuga Lange- } \\
\text { Bertalot }\end{array}$ & $\begin{array}{l}\text { Motile; motile } \\
\text { guild }\end{array}$ & $22-29$ & $4.7-6$ & $14-15$ & 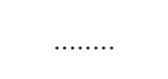 & \\
\hline $\begin{array}{l}\text { Navicula insulsa } \\
\text { Metzeltin \& Lange- } \\
\text { Bertalot }\end{array}$ & $\begin{array}{l}\text { Motile; motile } \\
\text { guild }\end{array}$ & $52.6-54.7$ & 7.4-7.9 & $14-16$ & 28 & \\
\hline $\begin{array}{c}\text { Navicula longicephala } \\
\text { Hustedt }\end{array}$ & $\begin{array}{l}\text { Motile; motile } \\
\text { guild }\end{array}$ & $16-21$ & $3.1-3.6$ & $18-20$ & ......... & \\
\hline $\begin{array}{l}\text { Navicula rostellata } \\
\text { Kützing }\end{array}$ & $\begin{array}{l}\text { Motile; motile } \\
\text { guild }\end{array}$ & $28-34$ & $7.8-9$ & $11-12$ & $\cdots \cdots \cdots$ & \\
\hline $\begin{array}{c}\text { Navicula salinicola } \\
\text { Hustedt }\end{array}$ & $\begin{array}{l}\text { Motile; motile } \\
\text { guild }\end{array}$ & $14.7-20$ & $2.6-3.6$ & $16-18$ & n....... & \\
\hline $\begin{array}{c}\text { Navicula symmetrica } \\
\text { R.M. Patrick }\end{array}$ & $\begin{array}{l}\text { Motile; motile } \\
\text { guild }\end{array}$ & $26.8-42$ & $5.7-7$ & $12-13$ & 22 & \\
\hline $\begin{array}{l}\text { Navicula veneta } \\
\text { Kützing }\end{array}$ & $\begin{array}{l}\text { Motile; motile } \\
\text { guild }\end{array}$ & $29-33$ & $6-7$ & $14-15$ & $\cdots \cdots \cdots$ & \\
\hline $\begin{array}{c}\text { Navicula vilaplanii } \\
\text { (Lange-Bertalot \& } \\
\text { Sabater) Lange-Bertalot } \\
\text { \& Sabater }\end{array}$ & $\begin{array}{l}\text { Motile; motile } \\
\text { guild }\end{array}$ & $12-15$ & 3 & $22-24$ & $\ldots \ldots \ldots$ & \\
\hline $\begin{array}{l}\text { Navigeia aikenensis } \\
\text { (R.M. Patrick) L.N. } \\
\text { Bukhtiyarova }\end{array}$ & $\begin{array}{l}\text { Motile; motile } \\
\text { guild }\end{array}$ & $10.5-28$ & $5.2-5.7$ & $12-13$ & -....... & \\
\hline $\begin{array}{c}\text { Navigeia lateropunctata } \\
\text { (J.H. Wallace) L.N. } \\
\text { Bukhtiyarova }\end{array}$ & $\begin{array}{l}\text { Motile; motile } \\
\text { guild }\end{array}$ & $18.4-26$ & $7.3-9$ & $15-16$ & ........ & \\
\hline \multicolumn{7}{|l|}{ Pleurosigmataceae } \\
\hline $\begin{array}{l}\text { Gyrosiga acuminatum } \\
\text { (Kützing) Rabenhorst }\end{array}$ & $\begin{array}{l}\text { Motile; motile } \\
\text { guild }\end{array}$ & $61-98$ & $9-11$ & $16-19$ & $19-21$ & \\
\hline $\begin{array}{l}\text { Gyrosigma scalproides } \\
\text { (Rabenhorst) Cleve }\end{array}$ & $\begin{array}{l}\text { Motile; motile } \\
\text { guild }\end{array}$ & $87-89$ & $13-14$ & 22 a 24 & ......... & \\
\hline \multicolumn{7}{|l|}{ Stauroneidaceae } \\
\hline $\begin{array}{l}\text { Stauroneis anceps } \\
\text { Ehrenberg }\end{array}$ & $\begin{array}{l}\text { Motile; motile } \\
\text { guild }\end{array}$ & $77-84$ & $14-16$ & $16-18$ & 18 & \\
\hline $\begin{array}{c}\text { Stauroneis thermicola } \\
\text { (J.B. Petersen) J.W.G. } \\
\text { Lund }\end{array}$ & $\begin{array}{l}\text { Motile; motile } \\
\text { guild }\end{array}$ & $11-14$ & $2-3$ & $20-24$ & $\cdots \cdots \cdots$ & \\
\hline
\end{tabular}


TABLE I (continuation)

\begin{tabular}{|c|c|c|c|c|c|c|}
\hline \multirow[b]{2}{*}{ Taxa } & \multirow[b]{2}{*}{$\begin{array}{l}\text { Growth form } \\
\text { and ecological } \\
\text { guild }\end{array}$} & \multicolumn{5}{|c|}{ Metric } \\
\hline & & Lenght $(\mu \mathrm{m})$ & Width $(\mu \mathrm{m})$ & Striae $(10 \mu \mathrm{m})$ & $\begin{array}{c}\text { Areolae }(10 \\
\mu \mathrm{m})\end{array}$ & $\begin{array}{c}\text { Fibulae/ } \\
\text { Costae/Alar } \\
\text { canals }(10 \\
\mu \mathrm{m})\end{array}$ \\
\hline \multicolumn{7}{|l|}{ Catenulaceae } \\
\hline $\begin{array}{l}\text { Halamphora montana } \\
\text { (Krasske) Levkov }\end{array}$ & $\begin{array}{l}\text { Motile; motile } \\
\text { guild }\end{array}$ & $14-15$ & $3-4$ & & & \\
\hline \multicolumn{7}{|l|}{ Nitzschiaceae } \\
\hline $\begin{array}{l}\text { Nitzschia acicularis } \\
\text { (Kützing) W. Smith }\end{array}$ & $\begin{array}{l}\text { Motile; motile } \\
\text { guild }\end{array}$ & $30-42$ & $3-4$ & $14-16$ & & \\
\hline $\begin{array}{l}\text { Nitzschia amphibia } \\
\text { Grunow }\end{array}$ & $\begin{array}{l}\text { Motile; motile } \\
\text { guild }\end{array}$ & $20-23$ & 4 & $13-14$ & & $6-7$ \\
\hline $\begin{array}{c}\text { Nitzschia clausii } \\
\text { Hantzsch }\end{array}$ & $\begin{array}{l}\text { Motile; motile } \\
\text { guild }\end{array}$ & $32-39$ & $3-4$ & $\ldots \ldots \ldots$ & $\ldots \ldots .$. & $8-10$ \\
\hline $\begin{array}{l}\text { Nitzschia frustulum } \\
\text { (Kützing) Grunow }\end{array}$ & $\begin{array}{l}\text { Motile; motile } \\
\text { guild }\end{array}$ & $22-28$ & $4-5$ & $20-24$ & $\ldots \ldots .$. & $9-11$ \\
\hline $\begin{array}{l}\text { Nitzschia linearis } \\
\text { (Agardh) W. Smith }\end{array}$ & $\begin{array}{l}\text { Motile; motile } \\
\text { guild }\end{array}$ & $62-66$ & 5 & & & $10-11$ \\
\hline $\begin{array}{l}\text { Nitzschia palea } \\
\text { (Kützing) W. Smith }\end{array}$ & $\begin{array}{l}\text { Motile; motile } \\
\text { guild }\end{array}$ & $36-48$ & $3-5$ & ........ & ......... & $12-14$ \\
\hline $\begin{array}{l}\text { Nitzchia terrestris (J.B. } \\
\text { Petersen) Hustedt }\end{array}$ & $\begin{array}{l}\text { Motile; motile } \\
\text { guild }\end{array}$ & $47-63$ & 4 & $\ldots \ldots \ldots$ & $\ldots \ldots \ldots$ & $5-7$ \\
\hline $\begin{array}{l}\text { Tryblionella debilis } \\
\text { Arnott ex O'Meara }\end{array}$ & $\begin{array}{l}\text { Motile; motile } \\
\text { guild }\end{array}$ & $16-27$ & $8-11$ & $17-19$ & ......... & $7-8$ \\
\hline \multicolumn{7}{|l|}{ Surirellaceae } \\
\hline $\begin{array}{c}\text { Surirella stalagma M.H. } \\
\text { Hohn \& J. Hellermann }\end{array}$ & $\begin{array}{l}\text { Motile; motile } \\
\text { guild }\end{array}$ & $14-16$ & $6-7$ & ......... & ......... & $6-7$ \\
\hline
\end{tabular}

The low-profile guild was composed of three species of the Achnanthidium Kützing complex. The low-profile guild abundance increased considerably (65\%) between after 1 and after 2 as a result of high rainfall ( $r=0.99 ; p=0.02)$. The high-profile guild diversity declined between after1 $\left(\mathrm{H}^{\prime}=2.44\right)$ and after2 ( $\left.\mathrm{H}^{\prime}=2.18\right)$ with a persistence of tube-forming diatoms. After1 and after 2 were marked by low Secchi depth (80-83 cm), high TS (100-90 mg.L $\left.{ }^{1}\right)$, and increases in rainfall $(85.2$ to $200 \mathrm{~mm})$. The high-profile guild diversity decreased substantially in $\operatorname{after} 3\left(\mathrm{H}^{\prime}=1.13 ; t=13.64 ; p<0.001\right)$ and was dominated by tube-forming diatoms.

PCA explained $82.99 \%$ of the variation in the dataset in the first two axes $(p<0.001)$ revealing a temporal gradient among diatom guilds, growth forms and the samples (Fig. 2). We used replicates as independent samples and along with the heteroscedasticity, the replicates of a given sampling period were plotted together. The first sample taken after the oil spill (after1) was characterized by the motile guild $(r=-0.71$; axis 2 ) comprised of motile diatoms ( $r=-0.98$; axis 2$)$. The after2 sample was characterized by the lowprofile guild ( $r=0.40$; axis 2$)$ and after3, by the high-profile guild ( $r=0.48$; axis 2 ). The after2 sample was comprised of erect (small and ruderal) taxa $(r=0.81$, axis 2$)$ and planktonic $(r=0.78$; axis 2) growth forms, whereas the after 3 sample was 


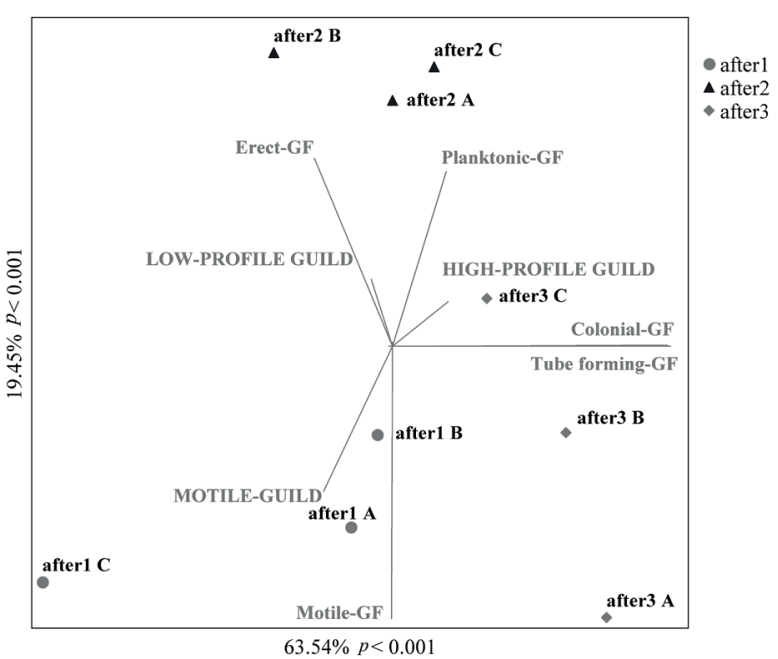

Figure 2 - Principal Component Analysis using diatom's guilds and growth forms (GF) showing temporal variation after the oil spill (A,B,C are the replicates from the same sampling date).

composed of colonial ( $r=0.99$; axis 1$)$ and tubeforming ( $r=0.99$; axis 1$)$ growth forms.

\section{DISCUSSION}

Vegetable oil forms a barrier on the surface water, constraining aquatic organisms by reducing dissolved oxygen and light availability. We registered high COD after the oil spill. COD in surface waters is useful for determining the degree of pollution by reflecting the total amount of hydrocarbon degradation. Thode-Filho et al. (2015) evaluated vegetable oil passive bioremediation (or natural attenuation) and revealed that the maximum biodegradation rate occurred around the thirtieth day of the experiment followed by total oxygen consumption. Reduced COD occurred in after3 and, with the high similarity among environmental variables, revealed a tendency to recover to the predisturbance conditions 24 days after the oil spill. Jézéquel et al. (2017) noticed high hydrocarbon degradation rates after one month under natural conditions. On the other hand, the authors also found a mortality rate of approximately $90 \%$ in the mangrove macrobenthic community exposed to hydrocarbon contamination.

The Negro River motile diatom guild was species-rich and dominated the diatom community after the oil spill. However, motile guilds in after 1 showed lower richness and diversity relative to the community sampled before the oil spill. The motile guild is formed by fast-moving species and strong competitors in nutrient-enriched environments; motile guild benefits from shaded places - when these species can move into the periphyton matrix as a response to this type of physical stress (Passy 2007). After1 was marked by high TS and shading, and high nutrients which may be due to the oil discharge (Snow and Scott 1975). Negative correlation between algae abundance and suspended matter was also observed by Ács and Kiss (1993) in the Danube River. Motile diatoms may become abundant in an environment with high concentrations of nutrients and TS (Faria et al. 2015). Notwithstanding the well-established literature around diatom guilds and growth forms, locating species tolerance information was difficult. Even with an accurate taxonomical investigation, we had some difficulties in finding infra-generic ecological data. Most of the Naviculaceae, Sellaphoraceae, and Diadesmidaceae taxa are undergoing constant taxonomical reviews and consequent synonymization and reclassification (Novais et al. 2013). Additionally, new taxa are continuously being discovered. The aforementioned taxa were recorded previously in polluted rivers (Lobo et al. 2004a, b, 2016, Hermany et al. 2006, Novais et al. 2013) and in an eutrophic reservoir (Marra et al. 2016), and are considered tolerant, in particular L. goeppertiana which has a high tolerance to TS (Céspedes-Vargas et al. 2016). Based on the literature, therefore, we considered them a tolerant taxa. The motile guild, having low diversity and being dominated by tolerant species, could be related to the disturbance caused by 
nutrients and shading, which sustains our biological traits approach.

Rainfall is a disturbing factor for periphytic communities which causes movement of inorganic particles and consequently shading by the drifted sediments (Ács and Kiss 1993). The high-profile guild comprises diatoms of tall stature, including stalked, filamentous, chain-forming, and tubeforming species that grow erect on substrates. These strategies provide capability to access resources as light and nutrients (Passy 2007). During their phylogenetic evolution, algae develop different strategies for attaching to surfaces or for surviving in flowing waters, after which the riverine periphyton can handle some constant disturbance level (Ács and Kiss 1993). Nevertheless, the high-profile guild becomes sensitive in light- and nutrient-rich environments (Berthon et al. 2011). Development of attached algae can be related to light penetration after the breaking of the oil barrier (Snow and Scott 1975), but we found very few changes in light penetration, maybe because the TS inputs were incited by the rainfall. However, the tube-forming Encyonema perpusillum (CleveEuler) D.G. Mann prevailed in after3 together with the low changes in light availability in the water column; their anatomic structure abets competitive ability for the use of light (Hermany et al. 2006). On the other hand, Rimet and Bouchez (2011) reported increases in tube-forming diatoms when testing pesticide-contamination effects in mesocosms. The authors hypothesized that the polysaccharide of the mucilaginous tube protected cells under chemical disturbances. We can argue, therefore, that tubeforming diatoms showed ability to compete for light and that their dominance over other growth forms could indicate chemical imbalance imposed on the diatom community, as well as reduction of available oxygen due to oil degradation.

The low-profile guild, mainly represented by Achnanthidium minutissimum (Kützing) Czarnecki, probably benefited from the environmental conditions that occurred in after2 due to high rainfall. The low-profile guild consists of short stature diatoms, slow-moving species, and solitary centrics (Passy 2007). The disturbance event favored pioneer growth forms and ruderal species (Peterson 1996) that are tightly attached to the substrate (Peterson and Stevenson 1992) and resistant after disturbances (Stevenson 1997, Schneck and Melo 2012) such as physical stress persisting in the periphytic matrix (Rimet and Bouchez 2012, Faria et al. 2015, Riato et al. 2017). A. minutissimum is largely recorded in lotic and lentic eutrophic environments (Lobo et al. 2004b, Ács et al. 2005), even in hypereutrophic conditions (Faria et al. 2013), and could be considered tolerant to strong nutrient inputs. The mean width of $A$. minutissimum valves was used as a biological trait related to nutrient levels in Swedish streams (Jarlman and Kahlert 2009, Kahlert et al. 2009). The authors proposed that mean width $<2 \mu \mathrm{m}$ is associated with oligotrophic waters, 2.2-2.8 $\mu \mathrm{m}$ with oligo-mesotrophic waters, and $>2.8 \mu \mathrm{m}$ with eutrophic waters. This trait applicability was tested and confirmed by Vilmi et al. (2015) who studied anthropogenic eutrophication of a large lake. The valve metrics of $A$. minutissimum from our study site showed larger widths (3-4 $\mu \mathrm{m})$ than observed by Vilmi et al. (2015). This data may support metric answers to nutrient inputs in the water or represent a local community response to natural conditions. Therefore, we encourage further investigations of morphological variation responses to chemical contamination and anthropogenic pollution.

Both diatom guilds and growth forms were useful for predicting the environmental conditions in the Negro River. While diatom guild richness formed a temporal gradient after the oil spill, the variability of the growth forms inside the guilds gave us tools to visualize the environmental conditions without accurate chemical monitoring. Changes inside the guilds were the main factor for understanding the environment. We point out that 
the high-profile and the motile guild diversities decreased over time due to increases in tubeforming diatoms and the resistance and dominance of the motile diatom $N$. aikenensis, respectively. These changes inside the guilds favoring the dominance of tolerant diatoms were strongly related to the responses of diatoms to abiotic changes largely presented in the literature. The disappearance of most diatoms in after 3 followed by the abovedescribed dominance may be the result of a combination of factors such as oil toxicity, low oxygen and disturbance-once the currents during raining period were able to remove dead cells. Since recolonization was not observed, we suspect that the oil density could be detrimental to the bioturbation activity, preventing natural propagule resuspension driven by the currents (Facca et al. 2002, Algarte et al. 2014).

Based on diatom functional traits, we were able to understand the environmental dynamics in a river driven by an unexpected oil spill. We were also able to find links between abiotic data, disturbances and biological traits. Therefore, we emphasize the importance of our findings in natural conditions. It is not possible to exclude the natural multistressors (such as canopy, differences in stream gradient and flow velocities, dispersal process, stochasticity, seasonality, differences in photoperiod, and/or grazers) in an uncontrolled field study. However, we highlight the importance of divulging our findings after this unexpected oil spill, as we have shown that environmental changes can be predicted by applying diatom biological tools. We believe that our results support the importance of continued investigation into diatom functional responses to physical and chemical disturbances. The use of ecological guilds is a valuable tool in monitoring environments after a disturbance because many taxa may use more than one strategy of attachment for competition or in handling physical stressors (Wang et al. 2014). Knowing diatom ecological preferences can abet their usefulness as a tool to assess environmental changes (Berthon et al. 2011) and better understand the dynamics and impacts occurring in a previously unstudied environment, as in the case of Negro River. We also support future research aimed towards applying controlled conditions to better understand diatom responses to oil contamination for possible future environmental predictions.

\section{ACKNOWLEDGMENTS}

The first author is grateful to the Coordenação de Aperfeiçoamento de Pessoal de Nível Superior (CAPES) and to Fundação Araucária for the concession of the Postdoctoral scholarship and also to the second author for the field efforts applied, as well as possible and without funding, when he noticed the oil spill during his Taxonomical Master degree. We thank the Conselho Nacional de Desenvolvimento Científico e Tecnológico (CNPq) for productivity grant to TAVL (308399/2016-1) and Cary Collet for the English review.

\section{AUTHOR CONTRIBUTIONS}

Denise M. de Faria designed the manuscript, produced the analyzes, results and discussion. Júlio C. Costin sampled, identified and counted the diatoms during his master degree. Priscila I. Tremarin identified the diatoms and furnished diatoms metrics. Thelma A.V. Ludwig rewiewed the text and contributed to the diatoms' identification, writing and discussion.

\section{REFERENCES}

ÁCS É AND KISS KT. 1993. Effects of the discharge on periphyton abundance and diversity in a large river (Danube, Hungary). Hydrobiol 249: 125-133.

ÁCS É, RESKÓNE NM, SZABÓ K, TABA G AND KISS KT. 2005. Application of epiphytic diatoms in water quality monitoring of Lake Velence - recommendations and assignments. Acta Bot Hung 3-4: 211-223.

ALGARTE VM, RODRIGUES L, LANDEIRO VL, SIQUEIRA T AND BINI M. 2014. Variance partitioning 
of deconstructed periphyton communities: does the use of biological traits matter? Hydrobiol 722: 279-290.

APHA. 2012. Standard methods for examination of water and waste water. 22nd ed., American Public Health Association (APHA), American Water Works Association (AWWA) and Water Environment Federation (WEF), Washington (DC), USA.

BAKKE T AND JOHNSEN TM.1979. Response of a subtidal sediment community to low levels of oil hydrocarbons in a Norwegian Fjord. In: Oil Spill Conference Proceedings 1979: 633-639.

BERTHON V, BOUCHEZ A AND RIMET F. 2011. Using diatom growth forms and ecological guilds to assess organic pollution and trophic level in rivers: a case study of rivers in south-eastern France. Hydrobiol 673: 259-271.

CÉSPEDES-VARGAS E, UMAÑA-VILLALOBOS G AND SILVA-BENAVIDES AM. 2016. Tolerancia de diez especies de diatomeas (Bacillariophyceae) a los factores físico-químicos del agua en el Río Sarapiquí, Costa Rica. Rev Biol Trop 64: 105-115.

CUBIT JD, GETTER CD, JACKSON JBC, GARRITY AD, CAFFEY HM, THOMPSON RCE, WEILS E AND MARSHALL MJ. 1987. An oil spill affecting coral reefs and mangroves on the Caribbean coast of Panama. In: International Oil Spill Conference Proceedings 1987: 401406.

DENICOLA DM AND KELLY M. 2014. Role of periphyton in ecological assessment of lakes. Fresh Sci 33: 619-638.

FACCA C, SFRISO A AND SOCAL G. 2002. Changes in abundance and composition of phytoplankton and microphytobenthos due to increased sediment fluxes in the Venice Lagoon, Italy. Estuar Coast Shelf Sci 54: 773-792.

FARIA DM, CARDOSO LS AND MOTTA-MARQUES D. 2015. Periphytic diatoms exhibit a longitudinal gradient in a large subtropical shallow lake. Inland Waters 5: 117-124.

FARIA DM, GUIMARÃES ATB AND LUDWIG TAV. 2013. Responses of periphytic diatoms to mechanical removal of Pistia stratiotes L. in a hypereutrophic subtropical reservoir: dynamics and tolerance. Braz J Biol 73: 681-689.

HAMMER Ø, HARPER DAT AND RYAN PD. 2001. PAST: Paleontological Statistics Software Software Package for Education and Data Analysis. Palaeontol Electronica 4: 1-9. Available at: https://palaeo-electronica.org/2001_1/ past/issue1_01.htm (accessed August 17, 2011).

HERMANY $\bar{G}$, SCHWARZBOLD A, LOBO EA AND OLIVEIRA MA. 2006. Ecology of the epilithic diatom community in a low-order stream of the Guaíba hydrographical region: subsidies to the environmental monitoring of the southern Brazilian aquatic systems. Acta Limnol Bras 18: 9-27.

HOFMANN G, LANGE-BERTALOT H AND WERUM M. 2013. Bestimmungflora Kieselalgen für die ökologische Praxis. Über 700 der häufigsten Arten und ihre Ökologie.
In: Lange-Bertalot H (Ed), Diatom Im Sübwasser-Benthos Von Mitteleuropa, $908 \mathrm{p}$.

JARLMAN A AND KAHLERT M. 2009. Påväxt I rinnande vatten - kiselalgsanalys, version 3:1. Handledning för miljöövervakning, Naturvårdsverket.

JÉZÉQUEL R ET AL. 2017. Assessment of oil weathering and impact in mangrove ecosystem: PRISME experiment. In: International Oil Spill Conference Proceedings 2017: 407.

KAHLERT M ET AL. 2009. Harmonization is more important than experience- results of the first Nordic-Baltic diatom intercalibration exercise 2007 (stream monitoring). J App Phycol 21: 471-482.

KOBAYASI H AND MAYAMA S. 1982. Most pollution tolerant diatoms of severely polluted rivers in the vicinity of Tokyo. Japan Jour Phycol 30: 88-196.

KRAMMER K. 1997a. Die cymbelloidean Diatomeen: eine Monographie der weltweit bekannten taxa. I Allgemeines und Encyonema part. Biblioth Diatomol 36: 1-382.

KRAMMER K. 1997b. Die cymbelloidean Diatomeen: eine Monographie der weltweit bekannten taxa. II Encyonema part., Encyonopsis and Cymbellopsis. Biblioth Diatomol 37: 1-469.

KRAMMER K AND LANGE-BERTALOT H. 1986. Bacillariophyceae: Naviculaceae. In: Ettl H, Gerloff J, Heynig H and Mollenhauer D (Eds), Süsswasserflora von Mitteleuropa Gustav Fischer, Jena. v. 2, pars 1, 876 p.

KRAMMER K AND LANGE-BERTALOT H. $1991 \mathrm{a}$. Bacillariophyceae: Centrales, Fragilariaceae, Eunotiaceae. In: Ettl H, Gerloff J, Heynig H and Mollenhauer D (Eds), Süsswasserflora von Mitteleuropa. Gustav Fischer, Stuttgart. v. 2, pars 3, 576 p.

KRAMMER K AND LANGE-BERTALOT H. $1991 \mathrm{~b}$. Bacillariophyceae: Achnanthaceae. Kritische Ergänzungen zu Navicula (Lineolatae) und Gomphonema. In: Ettl H, Gerloff J, Heynig H and Mollenhauer D (Eds), Gustav Fischer, Stuttgart. v. 2, pars 4, 437 p.

LANGE K, LIESS A, PIGGOTT JJ, TOWNSEND CR AND MATTHAEI D. 2011. Light, nutrients and grazing interact to determine stream diatom community composition and functional group structure. Fresh Biol 56: 164-278.

LEVKOV Z, LANGE-BERTALOT H, MITIĆ-KOPANJA D AND REICHARDT E. 2016. The Diatom Genus Gomphonema from the Republic of Macedonia: With 4425 Figures on 201 Plates. Koeltz Botanical Books.

LEVKOV Z, METZELTIN D AND PAVLOV A. 2013. Luticola and Luticolopsis. In: Lange-Bertalot H (Ed), Diatoms of Europe. Diatoms of the European inland waters and comparable habitats, Königstein: Koeltz Scientific Books 7: 1-698.

LOBO EA, BES D, TUDESQUE L AND ECTOR L. 2004a. Water quality assessment of the Pardinho river, RS, Brazil, using epilithic diatom assemblages and fecal coliforms as biological indicators. Vie Milieu 54: 115-125. 
LOBO EA, CALLEGARO VLM, HERMANY G, BES D, WETZEL CE AND OLIVEIRA MA. 2004b. Use of epilithic diatoms as bioindicators from lotic systems in southern Brazil, with special emphasis on eutrophication. Acta Lim Bras 16: 25-40.

LOBO EA, HEINRICH CG, SCHUCH M, DUPONT A, COSTA AB, WETZEL CE AND ECTOR L. 2016. Índice trofico de Qualidade da água. Guia ilustrado para sistemas lóticos subtropicais e temperados Brasileiros. Santa Cruz do Sul: EDUNISC, $57 \mathrm{p}$.

MARRA RC, TREMARIN PI, ALGARTE VM AND LUDWIG TV. 2016. Epiphytic diatoms (Diatomeae) from Piraquara II urban reservoir, Paraná state. Biot Neo 16: e201602000.

MCCUNE B AND MEFFORD MJ. 2011. PC-ORD Multivariate Analysis of Ecological Data.Version 6.0 MjM Software. Gleneden Beach, Oregon.

METZELTIN D AND LANGE-BERTALOT H. 1998. Tropical Diatoms of South America I. About 700 predominantly rarely known or new taxa representative of the neotropical flora. In: Lange-Bertalot H (Ed), Iconogr Diatomol, Annotated Diatom Monographs Gantner Verlag KG, Ruggell 5: 220.

METZELTIN D AND LANGE-BERTALOT H. 2007. Tropical Diatoms of south America II. Special remarks on biogeographic disjunction. In: Lange-Bertalot $\mathrm{H}$ (Ed), Iconogr Diatomol, Annotated Diatom Monographs, Gantner Verlag KG, Ruggell 18: 1876.

METZELTIN D, LANGE-BERTALOT H AND GARCÍARODRÍGUEZ F. 2005. Diatoms of Uruguay compared with other taxa from South America and elsewhere. In: LangeBertalot H (Ed), Iconogr Diatomol, Annotated Diatom Monographs, Gantner Verlag KG, Ruggell 15: 736.

MOREIRA-FILHO H AND VALENTE-MOREIRA IM. 1981. Avaliação taxonômica e ecológica das diatomáceas (Bacillariophyceae) epífitas em altas pluricelulares obtidas nos litorais dos Estados do Paraná, Santa Catarina e São Paulo. Bol Mus Bot Munic 47: 1-17.

NAYAR S, GOH BPL AND CHOU LM. 2004. The impact of petroleum hydrocarbons (diesel) on periphyton in an impacted tropical estuary based on in situ microcosms. J Ex Marine Biol Ecol 302: 213-232.

NOVAIS MH, WETZEL CE, VAN DE VIJVER B, MORAIS MM, HOFFMANN L AND ECTOR L. 2013. New species and combinations in the Geissleria (Bacillariophyceae). Cryptogamie, Algologie 34: 117-148.

PASSY SI. 2007. Diatom ecological guilds display distinct and predictable behavior along nutrient and disturbance gradients in running waters. Aquat Bot 86: 171-178.

PATRICK R AND REIMER CW. 1966. The Diatoms of United States: exclusive of Alaska and Hawaii. Acad Nat Sci, Philadelphia, Monografs 13: 688.
PETERSON CG. 1996. Response of benthical algal communities to natural physical disturbance. In: Stevenson RJ et al. (Eds), Algal ecology: freshwater benthic ecosystems, Academic Press, San Diego, CA, p. 375-402.

PETERSON CG AND STEVENSON JR. 1992. Resistance and resilience of lotic algal communities: importance of disturbance timing and current. Ecology 73: 1445-1461.

RIATO L, BELLA VD, LEIRA M, TAYLOR J AND OBERHOLSTER PJ. 2017. A diatom functional-based approach to asses changing environmental conditions in temporary depressional wetlands. Ecol Ind 78: 205-2013.

RIMET F AND BOUCHEZ A. 2011. Use of diatom growth form and ecological guilds to access pesticide contamination in rivers: Lotic mesocosm approaches. Ecol Indic 11: 489-499.

RIMET F AND BOUCHEZ A. 2012. Life-forms, cell-sizes and ecological guilds of diatoms in Europeans Rivers. Knowl Managt Aquatic Ecosyst 406: 1.

ROUND FE, CRAWFORD RM AND MANN DG. 1990. The Diatoms: biology and morphology of the genera. Cambridge (UK): Cambridge University Press.

SCHNECK F AND MELLO AS. 2012. Hydrological disturbance overrides the effect of substratum roughness on the resistance and resilience of stream benthic algae. Fresh Biol 57: 1678-1688.

SCHOLTEN M AND KUIPER J.1987. The effects of oil and chemically dispersed oil on natural phytoplankton communities. In: International Oil Spill Conference Proceedings 1987: 255-257.

SIMBERLOFF D AND DAYAN T. 1991. The guild concept and the structure of ecological communities. Annu Rev Ecol Evol Syst 22: 115-143.

SINGH AK AND GAUR JP. 1989. Algal epilithon and water quality of a stream receiving oil refinery effluent. Hydrobiol 184: 193-199.

SNOW NB AND SCOTT BF. 1975. The effect and fate of crude oil spilt on two Arctic lakes. In: International Oil Spill Conference Proceedings 1975: 527-534.

STENGER-KOVÁCS C, BUCZKÓ K, HAJNAL É AND PADISÁK J. 2007. Epiphytic, littoral diatoms as bioindicators of shallow lake trophic status: Trophic Diatom Index for Lakes (TDIL) developed in Hungary. Hydrobiol 589: 141-154.

STENGER-KOVÁCS C, LENGYEL E, CROSSETTI LO, ÜVEGES V AND PADISÁK J. 2013. Diatom ecological guilds as indicators of temporally changing stressors and disturbances in the small Torna-stream, Hungary. Ecol Ind 24: 138-147.

STEVENSON RJ. 1997. Scale-dependent determinants and consequences of benthic algal heterogeneity. J Nor Amer Benthol Soc 16: 248-262. 
STEVENSON RJ, BOTHWELL ML AND LOWE RL. 1996. Algae Ecology. San Diego: Academic Express.

SUDERHSA - SUPERINTENDENCIA DE DESENVOLVIMENTO DE RECURSOS HÍDRICOS E SANEAMENTO AMBIENTAL. 2007. Dados sobre a pluviosidade do rio Negro. Personal communication. mailto:edson-nagashima@suderhsa.pr.gov.br.14 de Março, 2007.

THODE-FILHO S, ALMEIDA TM, PAIXÃO CPS, MARQUES MRC AND SILVA ER. 2015. Passive bioremediation: a preliminary study on waste vegetable oil. Cienc Nat 37: 401-404.
VILMI A, KARJALAINEN SM, LANDEIRO VL AND HEINO J. 2015. Freshwater diatoms as environmental indicators: evaluating the effects of eutrophication using species morphology and biological indices. Environ Monit Assess 2015: 187-243.

WANG Q, HAMILTON PB AND KANG F. 2014. Observations on attachment strategies of periphytic diatoms in changing lotic systems (Ottawa, Canada). Nova Hedwigia 99: 239253.

WETZEL RG. 1983. Periphyton of freshwater ecosystems. The Hague: Dr. W. Junk Publishers. 